\title{
Colorimetric Multiplex Assay for Rapid Detection of Biogenic Amines
}

\author{
Eva-Maria Mayr $^{1}$, Andreas Zacherl ${ }^{1}$, Sara Doppler ${ }^{1}$, Claudia Preininger ${ }^{1}$ \\ ${ }^{1}$ AIT Austrian Institute of Technology, Konrad Lorenz Str. 24, 3430 Tulln, Austria \\ Claudia.preininger@ait.ac.at
}

\begin{abstract}
:
The consumption of food containing biogenic amines is reported to be responsible for many pharmacological effects (diarrhea, headache, asthma, hypotension, etc.) which lead to several types of foodborne disease, including histamine poisoning, mainly from ingestion of certain species of fish (scombroid poisoning), and tyramine toxicity (cheese reaction). Nevertheless, the occurrence of symptoms that result from such interactions remains hypothetical, because there are no acceptable clinical studies that demonstrate such reactions. One reason is the lack of reliable test methods.

In the project Bioamina we contribute to this issue developing a multiplex diagnostic tool that is capable of reliably and simultaneously measuring histamine, tyramine, putrescine and other biogenic amines in test food, serum and urine. Herein, we present first results on the isolation and characterization of new diamine oxidases and a proof-of-concept for biogenic amine detection via $\mathrm{H}_{2} \mathrm{O}_{2}$ production.
\end{abstract}

Key words: Enzyme, DAO, biogenic amine, histamine, putrescine

\section{Biogenic Amines in Food}

The consumption of food containing biogenic amines is reported to be responsible for many pharmacological effects which lead to several types of foodborne disease, including histamine poisoning, mainly from ingestion of certain species of fish (scombroid poisoning), and tyramine toxicity (cheese reaction). Amines have also been implicated as the cause of toxicity to chicks and were also studied as carcinogenic precursors. Nevertheless, opinions on the importance of biogenic amines, especially histamine are very contradictory, because there are no acceptable clinical studies that demonstrate a relation between the oral ingestion of biogenic amines and food intolerance reactions [1]. One reason is the lack of reliable test methods.

\section{Concept of the Multiplex Bioassay}

We therefore aim to develop a multiplex colorimetric bioassay consisting of several cross-reactive enzymes coupled with a pattern recognition algorithm to detect histamine, tyramine, putrescine, and other biogenic amines. The single enzyme does not identify a specific biogenic amine, but crossreacts with many biogenic amines to a certain extent.
Establishing a so-called crossreactivity array with a set of well-defined enzymes allows to identify and quantify a single biogenic amine as a result of the collective pattern of response to many biogenic amines. Neural network algorithms, a signal processing principle well suited to solve complex (large scale) problems which are currently intractable will be implemented using the "Neural Network" Toolbox from MATLAB for an optimized and reduced complexity of the data generated by the cross reactivity profile analysis. For data validation and quality control, real-time measurements will be compared with measurements performed on test samples.

\section{Newly Isolated Enzymes}

Diamine oxidases (DAO) have been isolated from various types of plants including (chick)peas, maize and barley using roots and shoots of seedlings and extracts of leaves [2].

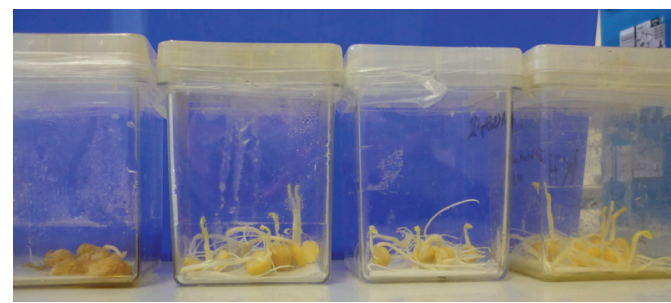


The extracts were purified by precipitation with ammonium sulfate and hydrophobic interaction chromatography. Enzyme activity using the guaiacol assay, storage stability at RT, 4 and $-20{ }^{\circ} \mathrm{C}$ and extraction yield were evaluated as well as the response profile towards histamine, putrescine, tyramine, spermine and spermidin to select the most appropriate enzymes for implementation in the multiplex assay.

\section{Colorimetric Detection}

Degradation of biogenic amines can be detected via the consumption of oxygen or the production of ammonia or hydrogen peroxide according to equation (1).

$$
\begin{aligned}
& \mathrm{R}-\mathrm{CH}_{2}-\mathrm{NH}_{2}+\mathrm{H}_{2} \mathrm{O}+\mathrm{O}_{2} \stackrel{\text { DAO }}{\longrightarrow} \mathrm{R}-\mathrm{CHO}+\mathrm{NH}_{3}+\mathrm{H}_{2} \mathrm{O}_{2} \\
& \mathrm{H}_{2} \mathrm{O}_{2}+\text { substrate } \stackrel{\text { HRP }}{\longrightarrow} \mathrm{H}_{2} \mathrm{O}+\text { chromogenic product }
\end{aligned}
$$

Our focus was on $\mathrm{H}_{2} \mathrm{O}_{2}$ detection using horseradish peroxidase (HRP) and tetramethylbenzidine (TMB) as enzyme substrate. Alternatively nanoparticles that mimic HRP behaviour were used. Details of the $\mathrm{H}_{2} \mathrm{O}_{2}$ sensors developed within this project will be presented at the conference.

\section{Outlook}

For validation of the colorimetric enzyme assays in clinically relevant samples an oral open food challenge study with healthy volunteers will be conducted at the Karl Landsteiner Institute St. Pölten in 2020.

\section{References}

[1] S.C. Jansen, M. van Dusseldorp, K. C. Bottema, and A. E. J. Dubois, Intolerance to dietary biogenic amines: a review, Annals of Allergy, Asthma \& Immunology 91, 233 -241 (2003); doi: 10.1016/S1081-1206(10)63523-5

[2] J. Lange, C. Wittmann, Enzyme sensor array for the determination of biogenic amines in food samples, Analytical Bioanalytical Chemistry 372, 276-283 (2002); doi: 10.1007/s00216-001-1130-9

\section{Acknowledgements}

This work is partly financed by the NÖ Forschungs- und Bildungsges.m.b.H. (NFB) within "Bioamina". 